IFIC/08-50

\title{
Modelling tri-bimaximal neutrino mixing
}

\author{
M. Hirsch,, ,* S. Morisi, ${ }^{1, \oplus}$ and J. W. F. Valle ${ }^{1, t}$ \\ ${ }^{1}$ AHEP Group, Institut de Física Corpuscular - C.S.I.C./Universitat de València \\ Edificio Institutos de Paterna, Apt 22085, E-46071 Valencia, Spain
}

(Dated: October 22, 2018)

\begin{abstract}
We model tri-bimaximal lepton mixing from first principles in a way that avoids the problem of the vacuum alignment characteristic of such models. This is achieved by using a softly broken $A_{4}$ symmetry realized with an isotriplet fermion, also triplet under $A_{4}$. No scalar $A_{4}$-triplet is introduced. This represents one possible realization of general schemes characterized by the minimal set of either three or five physical parameters. In the three parameter versions $m_{e e}$ vanishes, while in the five parameter schemes the absolute scale of neutrino mass, although not predicted, is related to the two Majorana phases. The model realization we discuss is potentially testable at the LHC through the peculiar leptonic decay patterns of the fermionic and scalar triplets.

PACS numbers: $11.30 . \mathrm{Hv} 14.60 .-\mathrm{z}$ 14.60.Pq 14.80.Cp
\end{abstract}

So far the historical discovery of neutrino oscillations constitutes the only indication of physics beyond the standard model. It is especially intriguing that the pattern of neutrino mixing angles [1] implied by current neutrino data is in sharp contrast with the structure of the Cabibbo-Kobayashi-Maskawa quark mixing matrix [2]. Current data do not yet fully determine the absolute scale of neutrino masses, nor do they shed any light on the important issue of leptonic $\mathrm{CP}$ violation [3, 4].

Lacking a basic theory of flavor and the origin of mass it is useful to have theoretical models restricting the pattern of fermion masses and mixings and providing guidance for future experimental searches. A successful phenomenological ansatz for leptons has been proposed by Harrison, Perkins and Scott (HPS) and is given by [5]

$$
U_{\mathrm{HPS}}=\left(\begin{array}{ccc}
\sqrt{2 / 3} & 1 / \sqrt{3} & 0 \\
-1 / \sqrt{6} & 1 / \sqrt{3} & -1 / \sqrt{2} \\
-1 / \sqrt{6} & 1 / \sqrt{3} & 1 / \sqrt{2}
\end{array}\right)
$$

which corresponds to

$$
\tan ^{2} \theta_{\mathrm{atm}}=1, \quad \sin ^{2} \theta_{\text {Chooz }}=0, \quad \tan ^{2} \theta_{\text {sol }}=0.5
$$

providing a good first approximation to the values indicated by current neutrino oscillation data.

In this note we discuss the general form of the effective neutrino mass operator which yields tri-bimaximal lepton mixing $U_{\text {HPS }}$. In general it is characterized by 5 physical parameters. These determine the two neutrino mass square differences measured in current neutrino os- cillation experiments. The third neutrino mass parameter, namely the absolute scale of neutrino masses, though not strictly predicted, correlates with the remaining two free parameters, namely two Majorana phases. Hence these would be indirectly tested by probing the absolute scale of neutrino mass in beta decay endpoint studies, neutrinoless double beta decay $(0 \nu \beta \beta)$ searches [6, 7], or cosmology 8].

We provide a possible gauge-theoretic realization based on the non-abelian discrete $A_{4}$ flavour symmetry. All $A_{4}$ models predicting $U_{\text {HPS }}$ lepton mixing, need at least two $A_{4}$-triplets whose vacuum expectation values (vevs) have different $A_{4}$-alignments. Different papers have emphasized this problem [9, 10, 11, 12]. In order to account for such alignments, extra-dimensions [13, 14] and/or supersymmetry [15, 16] have been invoked. Here we show how $U_{\text {HPS }}$ mixing can be obtained with just $A_{4}$ Higgs singlets in the framework of a softly broken $A_{4}$ theory. The model is similar in spirit, but inequivalent to the one suggested in Ref. [9].

We start by noting that if $M_{\nu}$ is

$$
M_{\nu}=\left(\begin{array}{ccc}
x(1+\alpha) & y(1+\alpha) & y(1+\alpha) \\
y(1+\alpha) & x+\alpha y & y+\alpha x \\
y(1+\alpha) & y+\alpha x & x+\alpha y
\end{array}\right)
$$

in the basis where charged leptons are diagonal, the lepton mixing matrix is $U_{\mathrm{HPS}}$ independently of the values of $x, y$ and $\alpha$, since it is $\mu \leftrightarrow \tau$ exchange invariant and $M_{\nu_{11}}=M_{\nu_{22}}+M_{\nu_{23}}-M_{\nu_{13}}$. Here $x, y$ and $\alpha$ are three 
complex free parameters. In such "weak-basis" the physical parameters can be chosen as their moduli $|x|,|y|,|\alpha|$, the phase of $\alpha$, which we denote with $\delta_{1}$ and the relative phase between $x$ and $y$, denoted as $\delta_{2}$. The eigenvalues of $M_{\nu}$ are

$$
\begin{aligned}
& m_{1}=(1+\alpha)(x-y), \\
& m_{2}=(1+\alpha)(x+2 y), \\
& m_{3}=(1-\alpha)(x-y) .
\end{aligned}
$$

Note that, the vanishing of $\theta_{13}$ removes the possibility of Dirac-type leptonic CP violation. Hence the two phases $\delta_{1}$ and $\delta_{2}$ are directly (but not trivially) related to the two physical Majorana phases of the lepton mixing matrix [17]. On the other hand we can rewrite the moduli $|x|,|y|,|\alpha|$ as the three neutrino mass parameters, which may be chosen as the two mass square splittings that enter the oscillations,

$$
\begin{aligned}
& \left|m_{3}\right|^{2}-\left|m_{1}\right|^{2}=\Delta m_{\text {atm }}^{2}= \\
& \quad=-4|\alpha| \cos \delta_{1}\left(|x|^{2}+|y|^{2}-2|x||y| \cos \delta_{2}\right), \\
& \quad\left|m_{2}\right|^{2}-\left|m_{1}\right|^{2}=\Delta m_{\text {sol }}^{2}= \\
& \quad=3\left(1+2|\alpha| \cos \delta_{1}+|\alpha|^{2}\right)\left(2|x||y| \cos \delta_{2}+|y|^{2}\right),
\end{aligned}
$$

plus the absolute mass scale that governs neutrinoless double beta decay

$$
\left|m_{e e}\right|^{2}=|x|^{2}\left(1+2|\alpha| \cos \delta_{1}+|\alpha|^{2}\right) .
$$

First we note that in the limit $|y|=0$ the neutrino mass matrix (2) is diagonalized by a maximal rotation in the 23 plane, but the "solar sector" is clearly inconsistent with experiment. On the other hand, when $|\alpha|=0$ the unitary diagonalizing matrix can not be fixed since there are two degenerate eigenvalues. Thus $|\alpha| \neq 0$ and $y \neq 0$ are required by experimental data. In contrast $|x|$ can be taken equal to zero, in fact in this case the three eigenvalues are distinct and the neutrino mass matrix $M_{\nu}$ is still diagonalized by the $U_{\text {HPS }}$ matrix. The most relevant phenomenological consequence of taking $|x|=0$ is that the neutrinoless double beta decay amplitude $\propto m_{e e}$ is zero, see Eq. (5).

We can solve the system in Eq. (4) with respect to $|x|$ and $|y|$ and write $m_{1,2,3}$ and $m_{e e}$ as function of $\delta_{1}, \delta_{2}$ and $|\alpha|$. For any value of $\cos \delta_{2}$, we have three possibilities: i) for $\cos \delta_{1} \sim 0$ we have $\left|m_{1}\right| \sim\left|m_{3}\right|$ that correspond

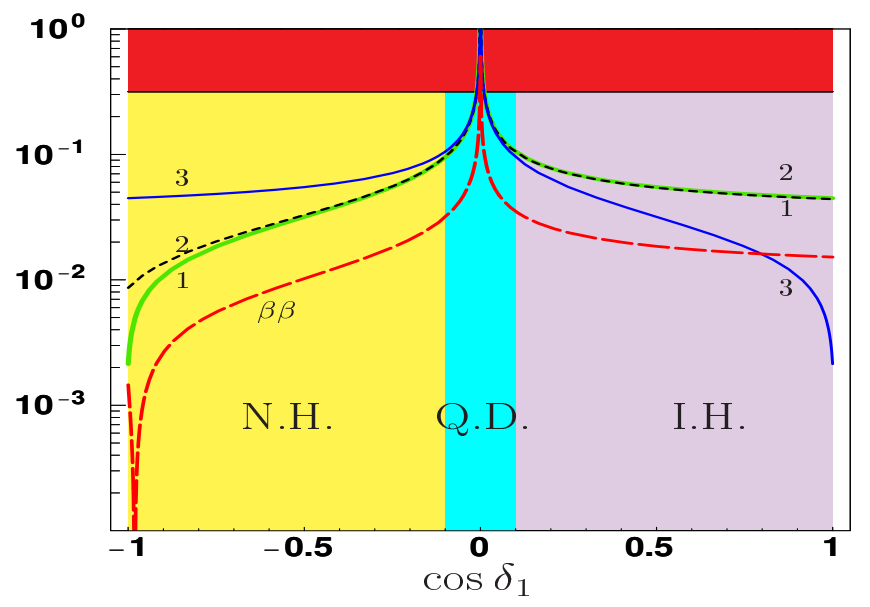

FIG. 1: $\left|m_{1}\right|$ (solid green), $\left|m_{2}\right|$ (small dashed dark), $\left|m_{3}\right|$ (solid blue) and $\left|m_{e e}\right|$ (large dashed red) vs $\cos \delta_{1}$ with $|\alpha|=$ 1.1 and $\cos \delta_{2}=1$.

to the degenerate spectrum, ii) if $\cos \delta_{1}<0$ we have $\left|m_{1}\right|<\left|m_{3}\right|$ or normal hierarchy and iii) if $\cos \delta_{1}>0$ we have $\left|m_{1}\right|>\left|m_{3}\right|$ or inverse hierarchy. These cases are illustrated in Fig. (1) where we plot the three masses versus $\cos \delta_{1}$ for a fixed value of $|\alpha|$ and $\cos \delta_{2}$. We note that for $\alpha \sim 0$ we have only the degenerate case since $\left|m_{1}\right| \sim\left|m_{3}\right|$.

The rate for neutrinoless double beta decay (5) vanishes when $|x|=0$. Solving the system in Eq. (4) for $|x|=0$ we find the relation

$$
\cos \delta_{1}=-\frac{3\left(1+|\alpha|^{2}\right)}{2|\alpha|(3+2 r)}
$$

where $r=\Delta m_{\text {sol }}^{2} / \Delta m_{\text {atm }}^{2}$. This special point correspond to the narrow dip in Fig. (11). By changing the value of $|\alpha|$ in Eq. (6) we have $-1 \leq \cos \delta_{1}<-0.975$ for $0.8<|\alpha|<1.24$ (we have used the best fit value for $r=0.032$, for different values the allowed range for $|\alpha|$ and $\cos \delta_{1}$ changes only slightly). For other values of $|\alpha|$ and $\cos \delta_{1}$ the neutrinoless double beta decay rate can not cancel. All the general considerations given here are quite independent of the value of $\cos \delta_{2}$ that is related to the difference between $\left|m_{e e}\right|$ and $\left|m_{1}\right|$. In fact if $\cos \delta_{2} \sim 0$ then $|y| \ll|x|$ and $\left|m_{e e}\right| \sim\left|m_{1}\right|$. We have taken $\cos \delta_{2}=1$ in Fig. (1) since it gives the lowest value of $m_{e e}$.

We now turn to a theoretical derivation of the neutrino 


\begin{tabular}{|c|cc|cc|}
\hline & $L_{i}$ & $l_{i}^{c}$ & $h_{1,2,3}$ & $\Delta_{s}$ \\
\hline$S U(2)$ & 2 & 1 & 2 & 3 \\
$A_{4}$ & 3 & 3 & $1,1^{\prime \prime}, 1^{\prime}$ & 1 \\
\hline
\end{tabular}

TABLE I: Lepton multiplet structure of our simple model, see text.

mass matrix in Eq. (2) based on the flavor symmetry $A_{4}$, the finite group of the even permutations of four objects (for a short introduction to $A_{4}$, see for instance [16] and references therein). It has four irreducible representations, one triplet 3 and three singlets $1,1^{\prime}, 1^{\prime \prime}$. Let $\chi$ and $\varphi$ be two triplets of $A_{4}$ then

$$
\begin{gathered}
1 \sim(\chi \varphi)=\left(\chi_{1} \varphi_{1}+\chi_{2} \varphi_{3}+\chi_{3} \varphi_{2}\right), \\
1^{\prime} \sim(\chi \varphi)^{\prime}=\left(\chi_{3} \varphi_{3}+\chi_{1} \varphi_{2}+\chi_{2} \varphi_{1}\right), \\
1^{\prime \prime} \sim(\chi \varphi)^{\prime \prime}=\left(\chi_{2} \varphi_{2}+\chi_{1} \varphi_{3}+\chi_{3} \varphi_{1}\right), \\
3_{s} \sim\left(\begin{array}{c}
2 \chi_{1} \varphi_{1}-\chi_{2} \varphi_{3}-\chi_{3} \varphi_{2} \\
2 \chi_{3} \varphi_{3}-\chi_{1} \varphi_{2}-\chi_{2} \varphi_{1} \\
2 \chi_{2} \varphi_{2}-\chi_{1} \varphi_{3}-\chi_{3} \varphi_{1}
\end{array}\right), 3_{a} \sim\left(\begin{array}{c}
\chi_{2} \varphi_{3}-\chi_{3} \varphi_{2} \\
\chi_{1} \varphi_{2}-\chi_{2} \varphi_{1} \\
\chi_{1} \varphi_{3}-\chi_{3} \varphi_{1}
\end{array}\right)
\end{gathered}
$$

and for instance [18]

$$
1 \sim(\chi \bar{\varphi})=\left(\chi_{1} \varphi_{1}+\chi_{2} \varphi_{2}+\chi_{3} \varphi_{3}\right)
$$

Consider as starting point the model defined in Table (II). We have three Higgs doublets $h_{1,2,3}$ in the $1,1^{\prime \prime}$ and $1^{\prime}$ representations of $A_{4}$ and one Higgs triplet $\Delta_{s}$ transforming as a singlet with respect to $A_{4}$. The $A_{4}$ invariant renormalizable Lagrangian reads

$$
\begin{aligned}
\mathcal{L}= & y_{1_{i j}}\left(L_{i} l_{j}^{c}\right) h_{1}+y_{2_{i j}}\left(L_{i} l_{j}^{c}\right)^{\prime} h_{2}+y_{3_{i j}}\left(L_{i} l_{j}^{c}\right)^{\prime \prime} h_{3}+ \\
& +y_{i j k}^{\Delta_{s}}\left(L_{i} L_{j}\right) \Delta_{s_{k}}
\end{aligned}
$$

where the structure of the Yukawa interaction can be easily obtained from the product rule in Eq. (7). Defining

$$
\left\langle h_{1}\right\rangle=v_{1}, \quad\left\langle h_{2}\right\rangle=v_{2}, \quad\left\langle h_{3}\right\rangle=v_{3}
$$

the charged lepton mass matrix is given as

$$
M_{l}=\left(\begin{array}{ccc}
a & b & c \\
b & c & a \\
c & a & b
\end{array}\right)
$$

where $a=y_{1} v_{1}, b=y_{2} v_{2}, c=y_{3} v_{3}$. It is diagonalized as

$$
\begin{gathered}
U_{\omega}^{\dagger} M_{l} U_{\omega}^{\dagger}=\left(\begin{array}{ccc}
a+b+c & 0 & 0 \\
0 & a+\omega b+\omega^{2} c & 0 \\
0 & 0 & a+\omega^{2} b+\omega c
\end{array}\right) \\
U_{\omega}=\left(\begin{array}{ccc}
1 & 1 & 1 \\
1 & \omega & \omega^{2} \\
1 & \omega^{2} & \omega
\end{array}\right)
\end{gathered}
$$

with $\omega^{3}=1$. As required, the charged lepton mass matrix has three distinct eigenvalues.

From the Lagrangian in Eq. (8) we have the tree level contribution to $M_{\nu}$ shown in Fig.(2):

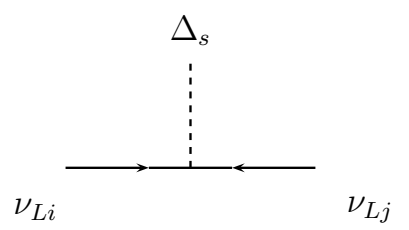

FIG. 2: Tree level contribution to $M_{\nu}$.

This diagram is the type-II seesaw [17] contribution to $M_{\nu}$. The tree-level diagram yields

$$
M_{\nu}^{0}=\left(\begin{array}{ccc}
z & 0 & 0 \\
0 & 0 & z \\
0 & z & 0
\end{array}\right)
$$

where $z=y^{\Delta_{s}} v_{s}$.

Consider now adding an $A_{4}$ triplet of fermion isotriplets $\Sigma \sim 3$ with hypercharge zero, together with one Higgs doublet being a singlet under $A_{4}, \eta \sim 1$. Both $\Sigma$ and $\eta$ carry an extra $Z_{2}$ quantum number. Since $\Sigma$ is in the adjoint representation of the electroweak group, it has a Majorana mass term, see for instance [19]. We have the following extra terms in the Lagrangian

$$
y_{\Sigma_{i k}}\left(L_{i} \Sigma_{k}\right) \eta+\mu_{k l} \Sigma_{k} \Sigma_{l}
$$

where we assume $\mu$ to break softly $A_{4}$, that is

$$
\mu=m_{\Sigma}\left(\begin{array}{lll}
1 & 0 & 0 \\
0 & \xi & 1 \\
0 & 1 & \xi
\end{array}\right)
$$

When $\xi \rightarrow 0, \mu$ respects $A_{4}$. In general the fermion triplets $\Sigma_{i}$ induce one-loop neutrino wave-function corrections, see Fig.(3), giving 


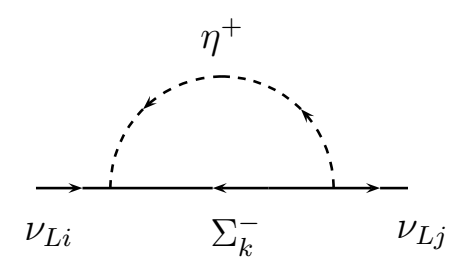

FIG. 3: One loop corrections to $M_{\nu}$ induced by fermion triplets $\Sigma_{i}$.

$$
R_{i j}=\frac{y_{\Sigma}^{2}}{16 \pi^{2}} \sum_{k} R_{i k}^{\mu} R_{j k}^{\mu *} B_{1}\left(m_{\Sigma_{k}}^{2}, m_{\eta}^{2}\right)
$$

where $m_{\Sigma_{1}}=m_{\Sigma}, m_{\Sigma_{2}}=m_{\Sigma}(-1+\xi), m_{\Sigma_{3}}=m_{\Sigma}(1+\xi)$ are the eigenvalues of the matrix $\mu$ in Eq. (14) and

$$
R^{\mu}=\left(\begin{array}{ccc}
1 & 0 & 0 \\
0 & 1 / \sqrt{2} & 1 / \sqrt{2} \\
0 & -1 / \sqrt{2} & 1 / \sqrt{2}
\end{array}\right)
$$

is the orthogonal matrix that diagonalizes $\mu$. Since the fermion triplet masses are not degenerate, the loop correction is off-diagonal,

$$
R=\left(\begin{array}{ccc}
\delta_{1} & 0 & 0 \\
0 & \delta_{2} & \delta_{3} \\
0 & \delta_{3} & \delta_{2}
\end{array}\right)
$$

with

$$
\begin{aligned}
\delta_{1} & =\frac{y_{\Sigma}^{2}}{16 \pi^{2}} B_{1}\left(m_{\Sigma_{1}}^{2}, m_{\eta}^{2}\right), \\
\delta_{2} & =\frac{y_{\Sigma}^{2}}{32 \pi^{2}}\left(B_{1}\left(m_{\Sigma_{2}}^{2}, m_{\eta}^{2}\right)+B_{1}\left(m_{\Sigma_{3}}^{2}, m_{\eta}^{2}\right)\right), \\
\delta_{3} & =\frac{y_{\Sigma}^{2}}{32 \pi^{2}}\left(B_{1}\left(m_{\Sigma_{3}}^{2}, m_{\eta}^{2}\right)-B_{1}\left(m_{\Sigma_{2}}^{2}, m_{\eta}^{2}\right)\right),
\end{aligned}
$$

The neutrino mass matrix $M_{\nu}^{0}$ in Eq. (12), is corrected by the one loop diagram Fig. (3) as follows

$$
M_{\nu}=M_{\nu}^{0}+R M_{\nu}^{0}+M_{\nu}^{0} R^{T}
$$

After some algebra Eqs. (11) and (18) are diagonalized by $U_{\text {HPS }}$ and $M_{\nu}$ is of the form of Eq. (2) as required.

If the $Z_{2}$ symmetry is broken, $\eta$ takes a vacuum expectation value $\langle\eta\rangle=u$ and the diagram of Fig. (44) gives a type-III seesaw contribution [20] to the neutrino mass matrix where the heavy Majorana neutrino of type-I seesaw is simply replaced with $\Sigma^{0}$ and is given by

$$
-y_{\Sigma} \cdot \mu^{-1} \cdot y_{\Sigma}^{T}
$$

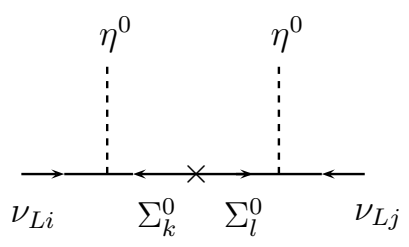

FIG. 4: Type III seesaw contribution to $M_{\nu}$.

Then from Eqs. (12) and (19) we have

$$
M_{\nu}=\left(\begin{array}{ccc}
z+\beta & 0 & 0 \\
0 & \frac{\beta \xi}{\xi^{2}-1} & z-\frac{\beta}{\xi^{2}-1} \\
0 & z-\frac{\beta}{\xi^{2}-1} & \frac{\beta \xi}{\xi^{2}-1}
\end{array}\right)
$$

where $\beta=y_{\Sigma}^{2} u^{2} / m_{\Sigma}$. Again $M_{\nu}$ is diagonalized by the $U_{\text {HPS }}$ in the basis where charged leptons are diagonal since in such a basis it has the form of matrix in Eq. (2) with the eigenvalues given by

$$
\begin{aligned}
& m_{1}=\frac{\beta+z(1+\xi)}{1+\xi} \\
& m_{2}=\beta+z \\
& m_{3}=\frac{\beta+z(1-\xi)}{1-\xi} .
\end{aligned}
$$

Eqs. (21) are compatible with normal, degenerate and inverse neutrino mass spectra. For instance when $\beta=$ $-z(1+\xi)$ then $m_{1}=0$ and we have the simple relation

$$
4 r=|1-\xi|^{2}
$$

If $\beta=-z(1-\xi)$ then $m_{3}=0$. This correspond to the inverse hierarchy and we have

$$
r=1-\frac{4}{|1-\xi|^{2}}
$$

In both cases we need $\xi \sim O(1)$ to reproduce data. For $|\xi| \ll 1$ we have degenerate neutrino masses.

We mention in passing that in general one could also have the situation where both tree-level, Fig. (4), and wave function correction, Fig. (3), contribute to $M_{\nu}$, leading however to the same overall structure in Eq. (2). We therefore do not discuss this "mixed" case in detail.

The above is just the simplest model with $U_{\text {HPS }}$ mixing that meets all phenomenological requirements. Other variants are easy to obtain. We could for example replace the Higgs triplet with two Higgs doublets. They contribute proportional to $z$ to the neutrino mass matrix via a dimension five operator. 
Finally we mention possible accelerator tests of our model realization. If the members of the scalar triplet are light enough to be produced at LHC [22], the decay pattern of the $\Delta_{s}^{++}$is completely fixed by the $A_{4}$ symmetry, and can be predicted to have only flavour diagonal decays $\operatorname{Br}\left(\Delta_{s}^{++} \rightarrow e^{+} e^{+}\right)=\operatorname{Br}\left(\Delta_{s}^{++} \rightarrow \mu^{+} \mu^{+}\right)=$ $\operatorname{Br}\left(\Delta_{s}^{++} \rightarrow \tau^{+} \tau^{+}\right)$with all $\operatorname{Br}\left(\Delta_{s}^{++} \rightarrow l_{i}^{+} l_{j}^{+}\right)=0$. This is different from, for example, the case discussed in [23], where the type-II seesaw is assumed to be the only source of neutrino mass. Also the cross section for the fermionic triplet at LHC has been recently studied [24, 25]. If $m_{\Sigma} \lesssim 1 T e V, \Sigma^{+} \Sigma^{-}$pairs will be produced decaying to $l^{+} l^{-}+2 \eta$. Again the branching ratios to the different lepton flavors are completely fixed by the $A_{4}$ symmetry. Pair produced $\Sigma^{ \pm}$will decay to two charged leptons plus two $\eta$ with branching ratios to the different lepton flavours approximately fixed by our ansatz for A4 breaking. Since the charged lepton mass matrix is diagonalized by $U_{\omega}$, the final states can be predicted to be $B r(e \mu) \simeq B r(e \tau), B r(\mu \mu) \simeq B r(\tau \tau)$ and $B r(e e) \simeq B r(\mu \mu)+B r(\mu \tau)-B r(e \tau)$.

In summary we have pointed out new ways to model $U_{\text {HPS }}$ lepton mixing from first principles. The minimal scheme is characterized by only three parameters and requires $m_{e e}=0$. Five parameter schemes can produce an arbitrary neutrino mass spectrum where $m_{e e}$ is not strictly predicted but related to the two Majorana phases of the lepton mixing matrix. We have discussed one model realization of these schemes, which interestingly avoids the vev misalignment problem present in other models based on $A_{4}$, as a consequence of not using $A_{4}$ triplet Higgses. This model is potentially testable at the LHC if either the scalar triplet or the fermionic triplet (or both) have mass below approximately $1 \mathrm{TeV}$.

\section{Acknowledgments}

We thank L. Lavoura and F. Bazzocchi for useful comments and discussions. Work supported by MEC grant FPA2008-00319/FPA, by European Commission Con- tracts MRTN-CT-2004-503369 and ILIAS/N6 RII3-CT2004-506222.

* Electronic address: mahirsch@ific.uv.es

$\dagger$ Electronic address: morisi@ific.uv.es

¥ Electronic address: valle@ific.uv.es

[1] T. Schwetz, M. Tortola and J. W. F. Valle, New J. Phys. 10, 113011 (2008), arXiv:0808.2016 [hep-ph]]; for previous analysis and relevant experimental references see also, e. g. M. Maltoni, T. Schwetz, M. A. Tortola and J. W. F. Valle, New J. Phys. 6, 122 (2004), hep-ph/0405172 v6]

[2] M. Kobayashi and T. Maskawa, Prog. Theor. Phys. 49, 652 (1973).

[3] H. Nunokawa, S. J. Parke and J. W. F. Valle, Prog. Part. Nucl. Phys. 60, 338 (2008), arXiv:0710.0554 [hep-ph]].

[4] ISS Physics Working Group, A. Bandyopadhyay et al., arXiv:0710.4947 [hep-ph].

[5] P. F. Harrison, D. H. Perkins and W. G. Scott, Phys. Lett. B530, 167 (2002), hep-ph/0202074.

[6] F. T. Avignone, S. R. Elliott and J. Engel, arXiv:0708.1033 [nucl-ex].

[7] M, Hirsch, arXiv:0609146 [hep-ph]

[8] J. Lesgourgues and S. Pastor, Phys. Rept. 429 (2006) 307 arXiv:astro-ph/0603494.

[9] E. Ma, arXiv:0808.1729 [hep-ph]

[10] W. Grimus and L. Lavoura, arXiv:0809.0226 [hep-ph]

[11] A. Zee, Phys. Lett. B630, 58 (2005), hep-ph/0508278.

[12] E. Ma, Phys. Rev. D70, 031901 (2004), hep-ph/0404199.

[13] G. Altarelli and F. Feruglio, Nucl. Phys. B720, 64 (2005), hep-ph/0504165.

[14] T. Kobayashi, Y. Omura and K. Yoshioka, arXiv:0809.3064 [hep-ph]

[15] K. S. Babu and X. G. He, arXiv:hep-ph/0507217

[16] G. Altarelli and F. Feruglio, Nucl. Phys. B741, 215 (2006), hep-ph/0512103.

[17] J. Schechter and J. W. F. Valle, Phys. Rev. D22, 2227 (1980).

[18] F. Feruglio, C Hagedorn, Y. Lin and L. Merlo, to appear.

[19] A. Abada, C. Biggio, F. Bonnet, M. B. Gavela and T. Hambye, Phys. Rev. D 78, 033007 (2008)

[20] R. Foot, H. Lew, X. G. He and G. C. Joshi, Z. Phys. C44, 441 (1989).

[21] E. Ma and D. Suematsu, arXiv:0809.0942 [hep-ph]

[22] J. F. Gunion, C. Loomis and K. T. Pitts, arXiv:9610237 [hep-ph]

[23] E. J. Chun, K. L. Lee, Y, Y. Kang and S. C. Park, Phys. Lett. B566, 142 (2003), arXiv:0304069 [hep-ph]

[24] R. Franceschini, T. Hambye and A. Strumia, Phys. Rev. D78, 033002 (2008), 0805.1613

[25] F. del Aguila and J. A. Aguilar-Saavedra, arXiv:0808.2468 